\section{Endoscopic vs Robotic Thyroidectomy: Which is Better?}

\section{TO THE EDITOR:}

We read with interest the article by Lee et al. ${ }^{1}$ and take this opportunity to congratulate them for their wonderful work. The authors compared the perioperative outcomes and learning curves of endoscopic (ET) and robotic thyroidectomy (RAT) in patients with differentiated thyroid cancer and found that the RAT had shorter total operation time, greater number of central lymph nodes retrieved, and shorter learning curve than ET. Coincidentally around the same time, we also reported our initial experience with these two procedures and compared perioperative outcomes between ET and RAT. However, in contrast to their findings, we found that RAT actually took significantly longer to complete than ET (149 vs. $100 \mathrm{~min}, P=0.018$ ), and this remained so even when similar extent of thyroid resection was compared. However, this seemingly contradictory finding could be explained by several fundamental differences between the two studies. Firstly, in our series, the majority of the patients had benign thyroid pathology and therefore did not require a central neck dissection (CND). We agreed that a CND is technically challenging endoscopically, and the availability of three robotic flexible endo-wrists in RAT certainly makes the procedure easier and shorter to complete. Secondly, Lee et al. had performed more than 80 ETs (which based on their own results, already well passed their learning curve) prior to their experience in RAT, whereas in our series, the surgeon who started RAT had fewer than 10 cases of ET experience. It is conceivable that extensive experience in ET could shorten the operating time and learning curve in RAT. In fact, to make the two procedures more comparable, our study deliberately compared our first seven cases of ET with the first seven cases of RAT, and yet the operation time was still significantly longer in RAT (120 vs. $149 \mathrm{~min}, P=0.004){ }^{2}$ In summary, we believe that unless a CND or a lateral neck dissection is required, for a straightforward hemithyroidectomy or total thyroidectomy, ET shortens the operation time while achieving similar perioperative outcomes. Furthermore, perhaps for surgeons who are contemplating starting their first few cases of RAT, it would be valuable to perform ETs initially (i.e., without the robot) so that they could be familiarized with the skin flap preparation and the lateral endoscopic view of the thyroid gland that are essential in RAT. However, to support our view, a future study could evaluate whether surgeons' prior experience in ET shortens the learning curve of RAT when compared with surgeons with no experience.

Brian Hung-Hin Lang, MS, FRACS, Man Po Chow, MBBS, MRCS, and Kai Pun Wong, MBBS, MRCS

Department of Surgery, The University of Hong Kong, Hong Kong SAR, China

e-mail: blang@hku.hk

Published Online: 10 June 2011

(C) The Author(s) 2011. This article is published with open access at Springerlink.com

OPEN ACCESS This article is distributed under the terms of the Creative Commons Attribution Noncommercial License which permits any noncommercial use, distribution, and reproduction in any medium, provided the original author(s) and source are credited.

\section{REFERENCES}

1. Lee J, Lee JH, Nah KY, Soh EY, Chung WY. Comparison of endoscopic and robotic thyroidectomy. Ann Surg Oncol. 2011;18: 1439-46.

2. Lang BH, Chow MP. A comparison of surgical outcomes between endoscopic and robotically assisted thyroidectomy: the authors' initial experience. Surg Endosc. 2011;25:1617-23. 